\title{
La dynamique de la politique européenne dans le
} Caucase

The European politics dynamics in Caucasus

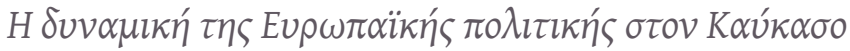

\section{Anastasia Ch. Lekka}

\section{(Q) OpenEdition}

\section{Journals}

Édition électronique

URL : https://journals.openedition.org/ceb/11704

DOI : 10.4000/ceb.11704

ISSN : 2261-4184

\section{Éditeur}

INALCO

Édition imprimée

ISBN : 978-2-85837-294-8

ISSN : 0290-7402

\section{Référence électronique}

Anastasia Ch. Lekka, «La dynamique de la politique européenne dans le Caucase », Cahiers

balkaniques [En ligne], 45 | 2018, mis en ligne le 05 décembre 2018, consulté le 08 juillet 2021. URL :

http://journals.openedition.org/ceb/11704 ; DOI : https://doi.org/10.4000/ceb.11704

Ce document a été généré automatiquement le 8 juillet 2021.

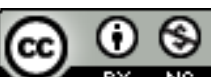

Cahiers balkaniques est mis à disposition selon les termes de la Licence Creative Commons Attribution - Pas d'Utilisation Commerciale 4.0 International. 


\title{
La dynamique de la politique européenne dans le Caucase
}

\author{
The European politics dynamics in Caucasus

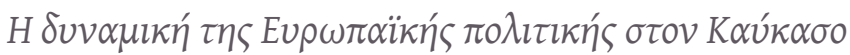

Anastasia Ch. Lekka

\section{Introduction}

1 Cet article se veut une introduction à l'étude de la nouvelle politique européenne dans le Caucase. L'étude des initiatives européennes régionales - politique européenne de voisinage, synergie de la mer Noire, partenariat oriental - cherche à promouvoir un dialogue scientifique autour de la stratégie européenne de coopération dans la région élargie du Caucase et des perspectives dans les relations de l'Union Européenne avec la Fédération de Russie et les États-Unis.

2 Dans la bibliographie internationale, l'étude de la Transcaucasie et les évolutions dans les pays de cette région ont constitué un objet de recherche sur un plan théorique et empirique depuis le début de la décennie 1990, avec pour principal point de référence les républiques de l'ex-Union Soviétique et les efforts pour établir des institutions politiques et économiques.

3 Nous avons la conviction que, bien que la région élargie du Caucase, aussi bien dans sa dimension historique que contemporaine, ait été examinée de façon exhaustive dans la bibliographie internationale, l'étude de la nouvelle politique européenne dans cette région peut apporter quelque chose de neuf au vu des évolutions qui ont lieu au début du XXI ${ }^{\mathrm{e}}$ siècle.

4 L'analyse de la politique européenne après la guerre froide en Europe du Sud-Est, dans la mer Noire et dans le Caucase ne relève pas, jusqu'à aujourd'hui, d'un acquis théorique et méthodologique accepté par tous les chercheurs. La présente étude se focalise sur l'interprétation des intérêts géopolitiques et géoéconomiques de la 
nouvelle stratégie européenne dans cette région, dans une approche réaliste, à travers le prisme de l'économie politique internationale.

5 Cet article essayera de répondre aux deux questions suivantes : quelles sont les raisons qui ont amené l'Union européenne à prendre des initiatives dans la région du Caucase, et pourquoi le Caucase constitue la région la plus instable de l'ex-Union soviétique.

\section{L'échiquier réaliste dans l'économie politique internationale}

6 On considère ici l'approche réaliste à travers le prisme de l'économie politique internationale, comme l'outil méthodologique approprié pour contribuer à mieux comprendre les changements économiques survenus dans le système régional au début du XXI ${ }^{e}$ siècle.

7 Le réalisme constitue l'ancienne école de pensée dans l'étude des relations internationales et il a permis d'analyser des sujets avec un grand succès. Cette théorie qui nous vient de Thucydide - le premier auteur réaliste -, est liée à une approche de l'économie politique internationale. Dans l'Histoire de la Guerre du Péloponnèse par exemple, Thucydide attribue la guerre entre les cités-États grecques à une série de changements économiques, dont l'augmentation du volume du commerce et l'émergence de nouvelles puissances commerciales comme Athènes et Corinthe. Il considérait la richesse comme une source de puissance militaire et estimait que la guerre n'est pas tant une affaire d'armes que d'argent ${ }^{1}$.

8 Dans cette approche théorique, nous tenterons de définir les composantes géoéconomiques et géopolitiques qui font du Caucase une région particulièrement importante aux yeux des acteurs internationaux et régionaux au début du xxi ${ }^{e}$ siècle.

9 Il est clair que les questions économiques ont toujours influencé la conjoncture internationale et déterminé le comportement des États; néanmoins, elles sont assurément de loin plus importantes depuis la fin de la guerre froide. Par ailleurs, dans les conditions de l'économie internationale, souligne Robert Gilpin, les États continuent d'user de leur autorité pour suivre des choix politiques profitables pour leurs intérêts et ceux de leurs citoyens ${ }^{2}$, parmi lesquels la jouissance de bénéfices ou de grandes parts de bénéfices provenant des activités économiques internationales, et la préservation de leur indépendance nationale ${ }^{3}$.

10 Un point-clé est que tous les États et toutes les régions qui produisent de grandes quantités de pétrole (à l'exception de l'Amérique du Nord) sont dans une situation instable, et pris dans différents conflits. L'Occident est certes actuellement moins dépendant du pétrole que par le passé, essentiellement en raison de l'adoption de nouvelles politiques énergétiques comme la création de stocks stratégiques de carburants ou la diversification des sources d'énergie en passant du pétrole à l'énergie nucléaire ou au gaz naturel, mais il reste que le pétrole continue à pouvoir provoquer une crise ${ }^{4}$.

11 Les objectifs de l'Occident -surtout des États-Unis - continuent d'être, entre autres, la stabilisation du prix international du pétrole brut au niveau le plus bas possible. Les États-Unis cherchent également à contrôler au maximum la production et le transport du pétrole et du gaz naturel vers les marchés occidentaux, en neutralisant les concurrents potentiels de l'Europe de l'ouest, de l'Orient (Russie et Chine) ou du 
Moyen-Orient. Dans ce contexte, l'Asie centrale et le Caucase, la mer Noire et les Balkans ont acquis une importance géostratégique particulière, soit en tant que régions productrices de pétrole et de gaz naturel, soit en tant que passages stratégiques pour le transport de ces produits. C'est ici qu'il faut chercher les raisons du nouveau jeu géoéconomique et géostratégique qui se joue après l'effondrement de l'Union soviétique. Plus spécifiquement, nous pouvons dire que les conflits qui ont lieu dans l'ensemble de la région sont en large part liés à l'exploitation du pétrole et du gaz. Lors de la guerre entre l'Arménie et l'Azerbaïdjan que les grandes puissances de la région ont largement attisée, la Russie a soutenu militairement et politiquement l'Arménie, tandis que l'Iran et la Turquie ont soutenu l'Azerbaïdjan. L'instabilité en Géorgie, qui vient des tendances séparatistes des républiques d'Abkhazie et d'Ossétie du Sud, tendances avivées par Moscou, s'explique par le rôle stratégique que joue l'Azerbaïdjan limitrophe dans l'acheminement du pétrole vers la Turquie. L'intervention russe en Tchétchénie est, elle aussi, liée au facteur pétrole ${ }^{5}$.

12 L'explosion du conflit entre la Russie et la Géorgie en août 2008 a constitué le sommet d'une guerre de faible intensité entre les deux États. Comme le signale Théodoros Tsakiris, « le pouvoir géorgien n'aurait jamais pu prendre la forme agressive qu'il a prise s'il n'avait pas pensé avoir le soutien des États-Unis et de l'OTAN $»^{6}$. Toutefois, l'aventure géorgienne n'est pas parvenue à bouleverser les relations entre les États-Unis et la Russie et assurément pas les relations entre Russie et Union européenne.

13 De son côté, la Russie cherche à renforcer son rôle régional et son rang international. Son changement essentiel au début $\mathrm{du} \mathrm{xxI}^{\mathrm{e}}$ siècle est que, tandis que jusque là elle semblait accepter la suprématie américaine dans d'importantes régions du monde, et se contentait de parlementer avec les Américains, elle cherche actuellement, en profitant de la conjoncture internationale, à exploiter le vide politique laissé par les politiques de Washington; en mettant en doute la suprématie américaine, dans un avenir plus éloigné, elle veut revenir de manière dynamique sur la scène internationale, comme l'un des protagonistes d'un système international supposé plus juste et plus sûr?

14 L'Union européenne a exprimé des inquiétudes similaires. Déjà dans Livre vert édité par la Commission européenne, on rappelle que le pétrole a une importance aussi vitale pour l'économie que le pain ${ }^{8}$. La famille européenne joue le premier rôle dans l'adoption de programmes énergétiques depuis la décennie 1990, en cherchant à en tirer bénéfice. L'un de ces programmes, le TACIS (Technical Aid to the Commonwealth of Independent States), comprend deux projets de réseaux d'infrastructures, le TRACECA (TRAnsport Corridor Europe Caucasus Asia, corridor de transport entre l'Europe, le Caucase et l'Asie) et l'INOGATE (INterstate Oil and GAs To Europe pipelines). Les réalistes, prétend Theodore Cohn, insistent sur les bénéfices qu'ils apporteraient et leur intérêt pour la survie et la sécurité des États9.

15 L'instabilité persistante dans la région du Caucase, et la conviction des dirigeants européens que seules la stabilité et la sécurité peuvent assurer des profits ont contribué à la prise d'importantes initiatives. Ces initiatives, telles qu'elles sont analysées ci-dessous, visent à assurer à long terme la sécurité énergétique et ses avantages. Par ailleurs, les nouvelles républiques du Caucase attendent, elles aussi, des profits des fonds et programmes européens pour l'amélioration des réseaux d'infrastructure dans des domaines tels que l'énergie, les transports, l'environnement, l'adoption de nouvelles connaissances technologiques, etc. 


\section{Politique européenne de voisinage}

16 défis nés du cinquième et du sixième élargissement en 2004 et en 2007. Un nouveau cadre pour les voisins orientaux et méridionaux de l'Europe élargie a été mis en œuvre pour la période 2007-2013, puis s'est poursuivi pour la période 2014-2020. Ses objectifs essentiels sont d'étendre les avantages de l'Europe des 27 aux pays voisins, en renforçant la sécurité, la stabilité et la prospérité dans la région. Cette politique vise également à lutter contre le risque d'apparition de nouvelles lignes de démarcation entre l'Union européenne et ses nouveaux voisins et à leur offrir la possibilité de participer au marché intérieur et à d'importantes activités européennes, par le biais de la coopération économique et sociale, en collaborant à des questions concernant la justice, les affaires intérieures, la lutte contre le crime organisé et l'immigration irrégulière ${ }^{10}$. régionale et elle ambitionne de contribuer à régler les conflits régionaux ${ }^{11}$. La promotion de la collaboration transfrontalière, avec la participation des autorités locales, régionales et des acteurs non gouvernementaux, devrait aider l'Union européenne à assurer la coopération et la coexistence pacifique dans les régions frontalières de l'Europe élargie. Cette politique encourage également les voisins de l'Union, qui ont signé des accords de coopération et de partenariat avec elle, à promouvoir des travaux d'infrastructure, surtout dans le domaine de l'énergie et d'autres domaines avec de nouvelles formes de coopération et d'interconnexion constructives $^{12}$.

Un des premiers pas dans la concrétisation de cette politique est le plan d'action déjà mis en place, un autre pas sera le début de négociations qui aboutiront à la conclusion des Accords européens de Voisinage qui remplaceront les accords bilatéraux existants. Cette politique s'adresse aux pays qui se trouvent à la bordure extérieure des frontières de l'UE à la suite de l'élargissement. Elle peut donc s'appliquer à l'Europe orientale, à la Biélorussie, à la Moldavie, à tous les pays de la Méditerranée - à l'exception de la Turquie, dont les relations avec l'UE sont liées aux procédures de préadhésion - et à la région du Caucase du sud (Arménie, Azerbaïdjan, Géorgie). À partir de 2007, elle s'étend aux pays de l'Europe orientale et de la mer Noire qui ont contracté avec l'Union des Accords de coopération et de partenariat. Les relations UE-Russie se développent dans quatre espaces communs de collaboration, définis lors du sommet de Saint-Pétersbourg en 2003, un espace économique commun, avec l'accent mis sur l'environnement et l'énergie, la coopération sur les questions de sécurité, de justice, dans le domaine de la sécurité extérieure ainsi que dans celui de la recherche et de l'éducation, héritage culturel compris ${ }^{13}$.

19 Le plan d'action couvre deux aspects importants pour l'UE: l'engagement dans des activités précises qui devront renforcer la cohésion concernant les valeurs et les objectifs communs dans le domaine de la politique étrangère et de la défense et, ensuite, une mise en œuvre qui rapprochera les pays voisins - partenaires de l'UE - de la famille européenne, par leur participation à une série de domaines prioritaires. Par exemple, les pays associés créeront une zone de libre-échange, en surmontant les obstacles des droits de douane et les problèmes techniques. 
20 Le plan d'action de la politique européenne de voisinage adopte comme moyen de financement l'Instrument européen de Partenariat et de Voisinage (IEPV), qui vient compléter les programmes déjà existants et se concentre sur des secteurs comme la collaboration transfrontalière, transnationale et régionale ; il est appliqué depuis 2007. En résumé, la politique européenne de voisinage constitue un cadre réaliste qui se fonde sur un ensemble commun de valeurs de base, adapté aux besoins concrets de chaque partenaire sur une base bilatérale. Les plans d'action lancés depuis 2004 concernent Israël, la Jordanie, le Maroc, la Moldavie, l'Autorité palestinienne, la Tunisie, l'Ukraine, et ils se sont étendus ensuite à l'Arménie, la Géorgie et l'Azerbaïdjan. Au-delà de la politique de voisinage, c'est la création d'un cercle de "pays amis" autour de l'UE ${ }^{14}$.

21 L'IEPV a mis à disposition, pour les nouvelles républiques du Caucase, la somme de 1034,1 millions d'euros pour la période 2007-2013 et a couvert une grande zone d'activités, comme le soutien au processus de démocratisation et de bonne gouvernance, à la mise en place de réformes pour améliorer l'administration, au développement des infrastructures et à la réduction de la pauvreté. Des sommes dont l'IEPV disposait pour cela durant la période 2007-2013, l'Ukraine est le pays qui a reçu la plus grande part (494 millions d'euros). Puis vinrent la Moldavie (209,7 millions d'euros), la Géorgie (120 millions d'euros), l'Arménie (98,4 millions d'euros), l'Azerbaïdjan (92 millions d'euros) et la Biélorussie (20 millions d'euros). Les domaines qui reçurent de l'IEPV les crédits les plus importants sont le développement économique et la modernisation des infrastructures ${ }^{15}$.

\section{Synergie de la mer Noire}

La région de la mer Noire a toujours constitué un nœud de communication pour le commerce mondial, un carrefour historique entre l'Occident et l'Orient, entre l'Europe et l'Asie, entre le Nord et le Sud. Les détroits du Bosphore relient les régions riches en matières premières de l'Asie centrale et de la Sibérie avec la Méditerranée, l'Europe et l'Afrique du Nord. C'est donc une voie de communication entre l'Europe et l'Asie centrale, ainsi qu'entre les marchés développés de la Chine et de l'Europe.

L'Organisation de Coopération économique de la mer Noire (OCEMN) fondée en 1992 à Istanbul comprend la Grèce, la Bulgarie, la Roumanie et la Moldavie (à l'ouest), l'Ukraine et la Russie (au nord), la Géorgie, l'Arménie et l'Azerbaïdjan (à l'est) et la Turquie (au sud). Bien que l'Arménie, l'Azerbaïdjan, la Moldavie et la Grèce n'aient pas de littoral sur la mer Noire, l'histoire, la proximité et les liens étroits qu'ils ont tissés en font des acteurs régionaux naturels. Les pays de la mer Noire ${ }^{16}$ forment un marché qui dépasse les 350 millions de consommateurs et ils ont en réserve un important capital industriel et humain, avec de sérieuses perspectives de développement.

La politique régionale européenne en mer Noire veut collaborer avec l'OCEMN pour pouvoir faire face aux défis et aux perspectives offertes aux deux parties. La CEMN avait poursuivi dès ses débuts un plan pour collaborer avec l'UE et s'intégrer dans le développement mondial, avec pour objectif principal de renforcer la stabilité, la sécurité et la prospérité dans les pays de la mer Noire à travers la coopération économique et le développement ${ }^{17}$. 
L'UE avait développé de nombreuses initiatives afin de soutenir les réformes économiques et politiques des pays ci-dessus, des accords bilatéraux, tels que le processus de préadhésion avec la Turquie, la politique de voisinage et la stratégie de partenariat avec la Fédération de Russie; toutefois, malgré un cadre bilatéral de relations avec chaque pays de la région, elle n'avait pas développé de relations directes avec la CEMN.

Les tentatives de rapprochement entre les pays de la mer Noire et l'UE ont débuté à Kiev le 14 février $2007^{18}$. La synergie de la mer Noire, comme a été appelée la nouvelle initiative de coopération, vise à développer des relations plus étroites d'une part entre les pays membres de l'Organisation de Coopération économique de la mer Noire, et d'autre part entre ces pays (en tant qu'ensemble unique) et l'UE. Cette nouvelle initiative régionale a été élaborée par la Commission européenne depuis 2007 dans le cadre de la politique européenne de voisinage avec des pays qui ne sont pas destinés à adhérer et elle se concentre sur la promotion de la coopération transfrontalière et régionale ${ }^{19}$. La Commission européenne participe à la synergie de la mer Noire en qualité d'observateur, ainsi que sept pays européens (Allemagne, France, Italie, Autriche, Pologne, Slovaquie et République de Tchéquie).

La coopération concerne un large rayon d'activités dans des domaines tels que l'infrastructure, l'énergie, l'environnement, les transports, le commerce, les déplacements de citoyens dans de bonnes conditions de sécurité, le renforcement des institutions démocratiques et de la bonne gouvernance, la recherche et la technologie, la promotion de la recherche officielle et des programmes d'éducation.

Un programme ambitieux signé par l'UE avec la Russie, en mars 2013, concerne la création d'un Marché énergétique paneuropéen qui doit se concrétiser d'ici $2050^{20}$. Le programme du réseau régional de transport de la mer Noire, quant à lui, s'est achevé en décembre 2013 et a soutenu le développement d'un réseau électrique dans le Caucase en renforçant le réseau électrique de transmission géorgien et en augmentant la capacité d'interconnexion entre la Géorgie et la Turquie. Un programme complémentaire qui vise à l'interconnexion entre la Géorgie et l'Arménie est actuellement à l'étude.

29 Le soutien financier de la nouvelle initiative s'appuie sur le principe de cofinancement de la part du programme communautaire de la politique de voisinage, de la Caisse de développement régional et d'autres programmes dont dispose l'UE, tout comme le soutien financier de la Banque européenne pour la Reconstruction et le Développement et de la Banque européenne d'Investissement.

La synergie de la mer Noire promeut le dialogue entre ces pays pour développer leur politique et leurs activités régionales. L'initiative vise également à mieux coordonner les projets et les programmes régionaux existants en leur apportant ainsi une valeur ajoutée et en évitant les doublons. De plus, elle met en place, dans des domaines spécifiques, de nouvelles dispositions législatives qui contribueront à renforcer le climat de confiance mutuelle entre les pays de la région.

31 Toutefois, l'expérience de ces dernières années met à l'épreuve la coopération régionale, dans un environnement composite et plus spécialement dans la situation géopolitique actuelle (le cas de l'Ukraine). La situation exige l'implication active d'un nombre toujours croissant d'acteurs, États membres de l'UE et pays membres de la mer Noire compris. 


\section{Partenariat oriental}

Le partenariat oriental est une nouvelle politique européenne d'inspiration suédoise et polonaise, inaugurée à Prague en 2009, qui s'adresse à l'Arménie, l'Azerbaïdjan, la Géorgie, la Moldavie, l'Ukraine et la Biélorussie ${ }^{21}$. Elle a été décidée en décembre 2008 à Bruxelles. Elle vise à apporter un soutien européen effectif et supplémentaire aux réformes dans les États de l'Europe orientale et du sud du Caucase en ce qui concerne la démocratie et l'économie de marché tout en renforçant leur intégrité étatique et territoriale. Cette stratégie européenne promeut la stabilité, la sécurité et la prospérité de l'UE, de ses partenaires, et du continent tout entier ${ }^{22}$.

Il s'agit d'une ambitieuse structure institutionnelle dans le cadre de la politique européenne de voisinage, dont on attend qu'elle réajuste son niveau d'engagement politique et qu'elle aide à contracter des accords bilatéraux d'association de nouvelle génération.

34 Les plates-formes officielles du programme sont:

- la démocratie, la bonne gouvernance et la stabilité ;

- l'intégration économique et la convergence avec les politiques européennes ;

- la sécurité énergétique ;

- les contacts entre les peuples.

Le partenariat oriental a donc pour objectif également une harmonisation avec l'économie européenne et la possibilité, pour les citoyens des pays ci-dessus, un voyage plus facile dans les pays européens, à condition de respecter les conditions de sécurité ; il instaure de nouveaux accords améliorés de sécurité énergétique qui profiteront à tous les pays contractants, ainsi qu'un soutien financier renforcée ${ }^{3}$.

Ce partenariat a été accueilli avec enthousiasme par les voisins de l'Est de l'UE, car il répond à leur souhait de se rapprocher davantage des institutions européennes d'intégration. L'UE, elle, a des intérêts vitaux dans la région où, depuis la fin de la guerre froide, des changements radicaux se sont opérés. Par ailleurs, les élargissements successifs ont renforcé la proximité de ces pays avec l'UE, comme l'avait souligné le président de la Commission européennen Jose Manuel Barroso; de surcroît, les réformes que soutient la politique européenne de voisinage ont déjà permis à ces pays de se rapprocher du système européen du point de vue politique et économique. L'UE veut resserrer ses relations avec ses voisins de l'Est, compte tenu du conflit en Géorgie en août 2008. Après le conflit dans le Caucase, le Conseil européen du 1er septembre a en effet invité la Commission à soumettre des propositions et à accélérer le processus de développement de la nouvelle initiative. Après avoir délibéré avec ses partenaires de l'Est, la Commission propose de développer des relations bilatérales plus profondes et de créer un nouveau cadre multilatéral de coopération. Les principaux nouveaux points de l'initiative sont les suivants ${ }^{24}$ :

- adoption de nouveaux accords d'association, dont des accords de libre-échange avec les partenaires qui sont prêts à prendre les engagements fermes que présupposent les accords d'association à l'égard de l'UE ;

- programmes globaux financés par l'UE afin d'améliorer la capacité administrative des partenaires;

- intégration progressive dans l'économie de l'Union européenne ; 
- encouragement des pays partenaires à développer un réseau de libre-échange entre eux, qui pourrait à long terme mener à la création d'une communauté économique de voisinage ;

- contraction de «pactes de mobilité et de sécurité » en vue de faciliter les voyages vers l'UE et, parallèlement, renforcement des efforts pour combattre la corruption, le crime organisé et l'immigration illégale. Les pactes devront également contribuer à la mise en conformité des structures d'octroi d'asile avec les normes de l'UE, dans le but plus éloigné d'abolir les visas d'entrée avec tous les partenaires qui y collaborent ;

- étude par la Commission des possibilités de mobilité de la main-d'œuvre dans le but de l'ouverture du marché européen du travail ;

- augmentation de la sécurité en matière d'énergie pour les partenaires européens et orientaux $^{25}$;

- programmes pilotes concernant le développement économique et social dans les pays partenaires, et visant plus spécialement à régler les disparités économiques et sociales existant entre ces pays ;

- création d'une politique multilatérale (plate-forme) dans quatre domaines: démocratie, bonne gouvernance et stabilité, intégration économique et convergence avec les politiques de l'UE, sécurité énergétique et contacts entre les peuples afin de renforcer les efforts de réforme entrepris par les pays partenaires ;

- initiatives dans des domaines à part de collaboration, comme la promotion de marchés régionaux d'électricité, le développement d'un nouveau corridor énergétique et la coopération en matière de prévention et de réponse aux catastrophes naturelles ou d'autre origine ;

- participation de la société civile et d'autres parties prenantes, Parlement européen compris ;

- aide financière supplémentaire ${ }^{26}$, à savoir, augmentation importante, passant de 450 millions d'euros en 2008 à 785 millions d'euros en 2013. L'aide présuppose un apport supplémentaire de 350 millions d'euros outre les ressources complémentaires pour la période entre 2010 et 2013. Est également prévue la répartition d'un montant de 250 millions d'euros qui a déjà été mis à disposition dans les programmes régionaux dans le cadre de la politique européenne de voisinage.

Cette structure institutionnelle du partenariat oriental, d'après la proposition de la Commission, doit faire preuve d'une souplesse d'adaptation ${ }^{27}$ aux besoins des différents partenaires. On propose donc des rencontres bisannuelles au sommet des chefs d'État et des gouvernements des 27 pays membres de l'UE et des six pays partenaires, pour décider d'une politique générale et d'actions concrètes. Le partenariat devrait également progresser par des réunions annuelles au printemps des ministres des Affaires étrangères, qui fournissent des orientations politiques et suivent les progrès dans l'application des décisions. Des rencontres de hauts fonctionnaires ont lieu au moins deux fois par an, préparées par la Commission européenne qui préside à leurs travaux en fonction des domaines concernés, selon les quatre plates-formes thématiques. Des groupes de travail spécialisés sont organisés à l'appui de ces quatre plates-formes thématiques, et se réunissent chaque fois que cela s'avère nécessaire. La collaboration à un niveau bilatéral se poursuit dans le cadre du programme de la politique européenne de voisinage.

Les relations du partenariat oriental avec les autres initiatives de coopération régionale en Europe sud-orientale, en mer Noire fonctionnent de manière complémentaire, mais leurs activités ne se recouvrent pas mutuellement. La synergie de la mer Noire a pour objectif de résoudre des problèmes qui exigent une participation des pays riverains et 
elle cherche à aligner les pays partenaires sur les institutions européennes d'intégration ; par conséquent, leur centre de gravité est Bruxelles ${ }^{28}$.

La Grèce, en tant que membre de la famille européenne, soutient le nouveau projet. Néanmoins, elle a formulé des réserves, en lien avec les inquiétudes sur sa politique intérieure, en insistant sur le fait qu'il ne faut pas négliger d'autres initiatives multilatérales régionales, tant au plan institutionnel qu'au plan économique, dans la mer Noire comme en Méditerranée.

40 Le partenariat oriental constitue une initiative encadrée et délimitée dans des domaines de collaboration bien précis et il ne se rapporte pas au développement de la coopération à un niveau plus général. Il a été inauguré officiellement en mai 2009 par une réunion au sommet des chefs d'États et des gouvernements des États membres de l'UE et des pays partenaires. Ce sommet spécial a adopté un texte politique dans lequel sont mentionnés les objectifs, les principes essentiels et les caractéristiques principales du processus de coopération qui résultera de ce partenariat.

\section{La nouvelle Stratégie européenne dans le Caucase. Relations UE - États-Unis - Russie}

41 L'UE s'est, de tout temps, intéressée au développement et au fonctionnement d'institutions politiques et économiques sur le modèle occidental dans la région voisine $\mathrm{du}$ Caucase. Les exaltations nationalistes, les tensions transfrontalières, les intérêts géostratégiques des États-Unis, de la Russie ainsi que d'acteurs régionaux émergents, ont pour conséquence que tous ces faits demeurent des plaies ouvertes au $\mathrm{XXI}^{\mathrm{e}}$ siècle, exactement comme durant la décennie $1990^{29}$.

Le conflit russo-géorgien d'août 2008 a montré une fois encore combien le Caucase est important dans les préoccupations géoéconomiques et géopolitiques de l'UE, des États-Unis et, bien sûr, de la Russie. Moscou a imposé son image de superpuissance régionale, en déclarant aux dirigeants et aux pays de son voisinage que c'est elle qui contrôle la région, et elle a également montré que l'Occident était incapable en pratique de soutenir les pays du Caucase en cas de crise grave. Le pouvoir russe, en août 2008, est parvenu à diviser l'Europe occidentale et a montré que les États-Unis ont besoin de ses services dans des domaines d'importance autrement plus vitale (comme l'Iran, la Syrie, l'Afghanistan) que les tensions de plus petite envergure avec des pays du Caucase $^{30}$.

43 La politique européenne, en août 2008, à l'égard de Moscou, a pu être qualifiée de modérée. L'utilité établie de la Russie pour la sécurité européenne, le volume de ses échanges commerciaux avec de nombreux États de l'UE et le besoin pour Bruxelles de s'éloigner un peu des États-Unis, ainsi que les besoins en énergie sont les paramètres essentiels qui ont incité un bon nombre de pays membres de l'UE à une certaine réserve en ce qui concerne l'adoption d'une position plus dure face à la Russie. L'UE se rend compte que ce n'est que grâce au développement de liens plus étroits avec la Russie qu'elle sera à même d'exercer une influence plus efficace sur elle. Parallèlement, elle attend de la Russie qu'elle montre une attitude responsable quant aux thèmes d'importance majeure qui touchent l'occident; en revanche, une marginalisation de Moscou, de toute évidence, la dégagerait de cette obligation de responsabilité et conduirait à amoindrir ses relations avec l'Occident, tandis que les corrélations 
intérieures se différencieraient au profit des cercles nationalistes et de l'ordre établi (surtout militaire) conservateur.

L'élection du président Obama a rendu clair le fait que les États-Unis tenteraient une approche globale avec les alliés européens en proposant le dialogue et la discussion au lieu d'entreprendre des démarches unilatérales. Par conséquent, on attendait entre les États-Unis et l'UE une collaboration plus étroite qui rendrait difficile l'émancipation de l'Europe par rapport à Washington ${ }^{31}$.

Une question d'importance vitale pour la sécurité et la stabilité de la région concerne la mer Caspienne. Son importance s'est accrue du fait que les intérêts géostratégiques de l'Occident, et en particulier des États-Unis, se consolideront davantage en cas de création de routes énergétiques qui contourneraient la Russie, limitant ainsi la dépendance de l'Europe par rapport à cette dernière. Par ailleurs, le projet et la construction de l'oléoduc Bakou-Tbilissi-Ceyhan constitue pour les États-Unis une priorité géopolitique essentielle ${ }^{32}$. À long terme, l'achat d'énergie et la nécessité pour la Russie de trouver des fournisseurs alternatifs constituent les éléments essentiels qui détermineront les évolutions ultérieures.

Toutefois, nous devons le signaler à propos de la Caspienne, les accords passés par la Russie avec le Kazakhstan (riche en pétrole), le Turkménistan (gaz naturel) et l'Ouzbékistan (important allié et fournisseur potentiel de gaz naturel), lui permettent de contrôler une grande partie de leurs exportations. Ainsi, Moscou a renforcé sa position par rapport à l'Occident et limite les possibilités des États en question de contracter un accord direct de fourniture d'énergie avec des pays tiers, sans sa participation ou son consentement.

\section{Conclusion}

Les priorités économiques de l'UE dans la région élargie du Caucase sont liées aux questions énergétiques qui sont devenues particulièrement vives après 2005. L'augmentation de la demande en pétrole et en gaz naturel, les questions soulevées au sujet de formes d'énergie propre, pour faire face aux problèmes environnementaux, la lutte russo-ukrainienne (janvier 2006) pour le gaz naturel et le conflit russo-géorgien (août 2008) ont fonctionné comme des catalyseurs et ont clairement mis en évidence la dépendance énergétique des États européens et la nécessité pour eux de réagir.

Les régions riches en ressources énergétiques du Moyen-Orient, d'Asie centrale, du Caucase possèdent d'importants stocks de pétrole et de gaz naturel, et, en même temps, concentrent la concurrence internationale. L'effondrement de l'Union soviétique a contribué à révéler les nouveaux producteurs en mer Caspienne, tandis que les grands acteurs de la région élargie qui soit produisent de l'énergie soit tentent de contrôler les conduites de transport du pétrole et du gaz naturel demeurent la Russie, l'Iran et bien sûr les États-Unis.

Les opportunités géoéconomiques plus vastes composent une diplomatie européenne dynamique qui n'est pas dictée par des intérêts permanents dans la région. L'UE, en essayant de réagir à cet environnement international anarchique et concurrentiel, a pris une série d'initiatives, telles que la politique européenne de voisinage, la synergie de la mer Noire et le partenariat oriental, dans le but de diversifier sa dépendance de sources, de ressources et de routes énergétiques ainsi que d'assurer son bon 
approvisionnement. Elle a compris en effet que son approvisionnement énergétique est étroitement lié à la stabilité, la démocratisation et le développement économique de la région sensible du Caucase.

\section{BIBLIOGRAPHIE}

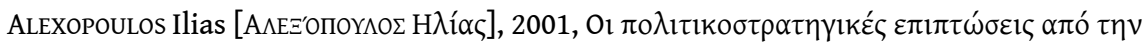

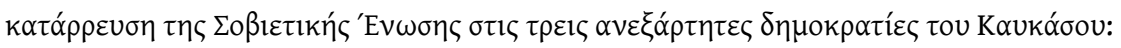

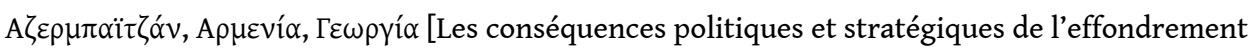
de l'Union soviétique dans les trois républiques du Caucase : Azerbaïdjan, Arménie, Géorgie],

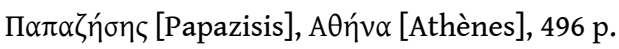

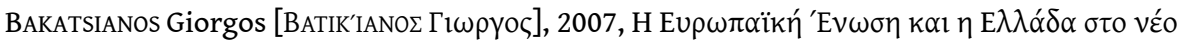
$\pi \alpha \gamma \kappa o ́ \sigma \mu 10 ~ \pi \varepsilon \rho ı \alpha ́ \alpha \lambda$ ov [L’Union européenne et la Grèce dans le nouveau contexte mondial],

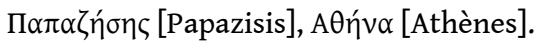

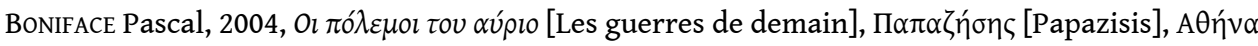
[Athènes], $270 \mathrm{p}$.

CoHn Theodore H., 2000, Global Political Economy, Theory and Practice, Pearson-Longman, Harlow,

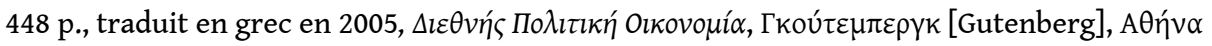
[Athènes].

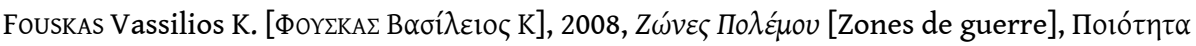
[Piotita], AӨńv $\alpha$ [Athènes], 336 p.

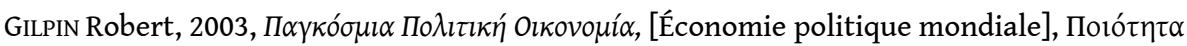
[Piotita], AӨńva [Athènes], $525 \mathrm{p}$

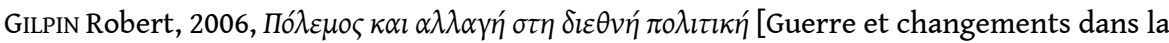

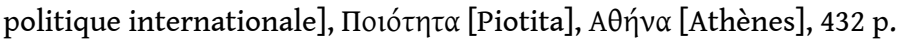

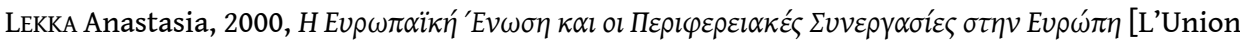

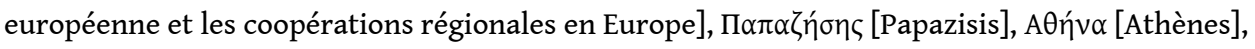
$544 \mathrm{p}$.

MONTEN Jonathan, 2006, “Thucydides and Modern Realism”, International Studies Quarterly 50/1, pp. 3-25.

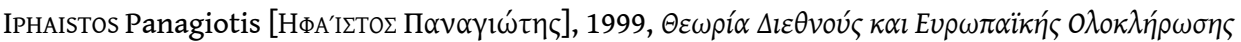

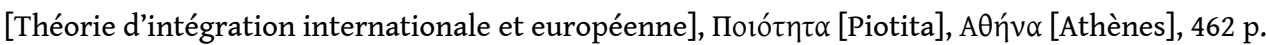

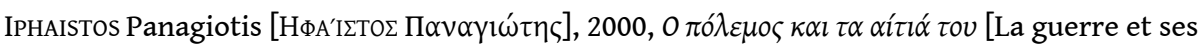

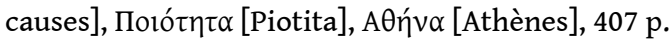

PHILIS Konstantinos, 2008, « Enseignements à tirer de la crise récente dans le Caucase », Agora without Frontier, p. 13.

TSAKIRIS Theodoros, 2008, « La guerre froide inachevée : Préhistoire, Chronique de la guerre russo-géorgienne ", Agora without Frontier, vol. 14/1, p. 14. 
YANNIS Alexandros, 2008, “The European Union and the Black Sea region. The new eastern frontiers and Europeanisation", International Centre for Black Sea Studies, ICBSS, Athens, Policy Brief no 7.

\section{Documents européens}

European Commission Document. The EU's relations with Russian 17.05.2001. http// europa.eu.int/comm/externalrelations/russia/sum.mit17.05.01/statementhtm.

European Commission. Communication from the Commission Paving the way for a New Neighborhood Instrument COM (2003) 293 Final 1.7.2003, et European Commission. Communication from the Commission. A Strong European Neighbourhood Policy, COM (2007) 774 Final, Brussels 05/12/2007. http://www.consilium.europa.eu/en/press/press-releases/ 2015/04/20-council-conclusions-review-european-neighbourhood-policy, consulté le 2 mai 2017. Council Conclusions review 20/4/2015. European Commission COM (2007) 774 Final, Brussels, 05/12/2007.

European Commission. Communication from Commission in the Council and European Parliament. Progress report on implementation of the European Neighbourhood Policy, sectoral progress, COM (2009) 188/4, Brussels 23/04/2009.

www.enpi-info.eu/ENIEuropean-Neighbourhood-instrument.

Country Strategy Paper 2007-2013 and National Indicative Programma 2007-2013.

"Opinion of the Committee of the regions and Black Sea Synergy - A new regional Cooperation initiative", Official Journal of the European Union (2008/C 105/10), 25.4.2008, and document of the European Commission BSEC-EU Interaction. The BSEC approach", Brussels, 2006.

"The joint Statement of the Ministers of Foreign Affairs of the Countries and the EU and of the Wider Black Sea Area, Kyiv 14 February 2008" et "European Communication - communication from the commission to the European Parliament: Black Sea Synergy - A new regional cooperation initiative" COM (2007) 160 Final, Brussels, 11 April 2007.

"Black Sea Regional Strategy 2009-2013. Promoting a region-wide interaction between BSEC and EU", 2008, Working Paper submitted by the ICBSS to the ad hoc Group of Experts on BSEC-EU interaction, Athens, 26 September.

European Commission, Communication from the Commission to the Council and the European Parliament, Eastern Partnership, COM (2008) 823 Final, Brussels, 31 December 2008, p. 3, ainsi que : Commission Staff Working Document accompanying the Eastern Partnership, Brussels, SEC (2008) $297 / 4 / 3$.

Eastern Partnership, Communication 31 December 2008.

"Black Sea Synergy review of a regional cooperation initiative", European Commission, High Representative of EU for foreign Affairs and Security Policy. Brussels 21.1.2015, SWD (2015) 6 Final.

European Commission about Eastern Partnership, questions and answers, et Eastern Partnership Communication 31 December 2008, (unités thématiques 3 et 4).

European Neighborhood Policy (Action Plan), “Eastern Partnership - an ambitious project for $21^{\text {st }}$ century European Foreign Policy", by Commissioner for external relations and ENP. 


\section{NOTES}

1. CoHN, 2005, p. 103 ; GILPIN, 2003, p. 105, et du même auteur, 2006, p. 125 ; MonTEN, 2006, p. 3-25.

2. IPHAISTOS, 1999, p. 332.

3. GILPIN, 2003, p. 36.

4. FOUSKAS, 2008, p. 76-77, IPHAISTOS, 2000, p. 359. P. Iphaistos, traitant des causes de la guerre, soutient que «la quête de nouvelles ressources ou de ressources susceptibles d'offrir des bénéfices constitue, entre autres, une cause de guerre dans le monde actuel ».

5. BONIFACE, 2004, p. 144.

6. TSAKIRIS, 2008, p. 14.

7. PHILIS, 2008, vol. 14/1, p. 13.

8. FOUSKAS, 2008, op. cit., p. 73.

9. COHN, 2005, p. 126. GILPIN, 2003, p. 125-126.

10. http://www.consilium.europa.eu/en/press/press-releases/

2015/04/20-council-conclusions-review-european-neighbourhood-policy. European Commission. Communication from the Commission Paving the way for a New Neighbourhood Instrument COM (2003) 293 Final 1.7.2003, et European Commission. Communication from the Commission: A Strong European Neighbourhood Policy, COM (2007) 774 Final, Brussels 05/12/2007, consulté le 2 mai 2017.

11. Council Conclusions review 20/4/2015. European Commission COM (2007) 774 Final, Brussels, 05/12/2007, p. 6.

12. European Commission. Communication from Commission in the Council and European Parliament. Progress report on implementation of the European Neighbourhood Policy, sectoral progress, COM (2009) 188/4, Brussels 23/04/2009.

13. Nous rappelons que la collaboration entre l'UE et la Russie a été mise en route depuis la conclusion des Accords de Partenariat et de Coopération des deux parties impliquées. Voir European Commission Document, The EU's relations with Russian 17.05.2001. http//europa.eu.int/comm/externalrelations/russia/sum.mit17.05.01/ statementhtm.

14. L'IEPV a remplacé l'EPV pour la période 2014-2020 avec un nouveau budget de 15,4 billions d'euros. Voir www.enpi-info.eu/ENIEuropean-Neighbourhood-instrument.

15. European Commission Assistance programming documents: ENPI Armenia, Azerbaijan, Belarus, Georgia, Moldova, Ukraine, Country Strategy Paper 2007-2013 and National Indicative Programma 2007-2013.

16. Voir LEKKA, 2000, p. 181-182.

17. "Opinion of the Committee of the regions and Black Sea Synergy - A new regional Cooperation initiative», Official Journal of the European Union (2008/C 105/10), 25.4.2008, and document of the European Commission BSEC-EU Interaction. The BSEC approach", Brussels, 2006, pp. 3-4. Voir également Alexandros YANNIS, 2008, The European Union and the Black Sea region: The new eastern frontiers and europeanisation, International Centre for Black Sea Studies - AӨńva: ICBSS, Policy Brief no 7, p. 4. 
18. "The joint Statement of the Ministers of Foreign Affairs of the Countries and the EU and of the Wider Black Sea Area, Kiev, 14 February 2008" et "European Communication - communication from the commission to the European Parliament: Black Sea Synergy - A new regional cooperation initiative" COM (2007) 160 Final, Brussels, 11 April 2007.

19. "Black Sea Regional Strategy 2009-2013. Promoting a region - wide interaction between BSEC and EU", 2008, Working Paper submitted by the ICBSS to the ad hoc Group of Experts on BSEC-EU interaction, Athens, 26 September, pp. 2-3.

20. "Black Sea Synergy review of a regional cooperation initiative", European Commission, High Representative of EU for foreign Affairs and Security Policy. Brussels 21.1.2015, SWD (2015) 6 Final, p. 4.

21. La participation de la Biélorussie au partenariat oriental était liée à l'évolution globale de ses relations avec l'UE.

22. European Commission, Communication from the Commission to the Council and the European Parliament, Eastern Partnership, COM (2008) 823 Final, Brussels, 31 December 2008, p. 3 ainsi que Commission Staff Working Document accompanying the Eastern Partnership, Brussels, SEC (2008) 297/4/3.

23. Eastern Partnership, Communication 31 December 2008, p. 8.

24. European Commission about Eastern Partnership, questions and answers, p. 3 et Eastern Partnership Communication 31 December 2008, p. 4 et 11 (unités thématiques 3 et 4).

25. European Neighbourhood Policy (Action Plan), “Eastern Partnership - an ambitious project for $21^{\text {st }}$ century European Foreign Policy", by Commissioner for external relations and ENP.

26. European Commission Document, p. 4.

27. European Commission Document, p. 6.

28. European Commission document, pp. 7-8 et Yannis T SANTOULIS, 2009, Black Sea Synergy and Eastern Partnership Different Centers of Gravity Complementary or Confusing Signals? International Centre for Black Sea Studies, Athens, ICBSS, 12 February 2009.

29. Alexopoulos, 2001, p. 96.

30. PHILIS, 2008.

31. Ibid., p. 10.

32. BAKATSIANOS, 2007, p. 83.

\section{RÉSUMÉS}

Cet article étudie la politique européenne - initiatives régionales - dans la région élargie du Caucase au vu des évolutions qui ont lieu au début du Xxi ${ }^{e}$ siècle.

Les intérêts géopolitiques et géoéconomiques dans le système de relations Union Européenne-EU, UE.-Fédération de Russie, EU-Fédération de Russie constituent l'objet de la recherche dans la 
région examinée (Europe du Sud-Est, Mer Noire) à travers le prisme de l'approche réaliste dans le cadre de l'économie politique internationale.

This paper deals with the European policy -European regional initiatives- in the Greater Caucasus region according to developments at the beginning of the $21^{\text {st }}$ century. Geopolitical and geo-economic interests in the European Union relations system USA-EU-Russian Federation, USA- Russian Federation, constitute the object of the research in the region examined (South-East Europe, Black Sea) through the prism of a realistic approach within the framework of the international political economy.

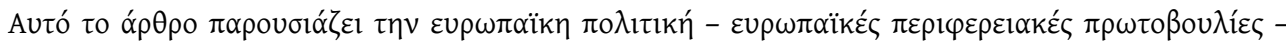

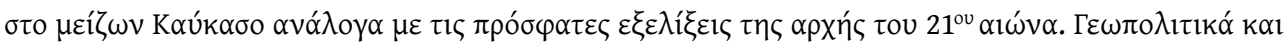

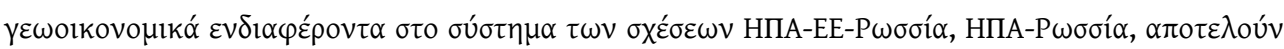

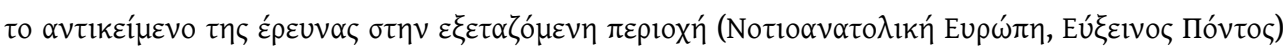

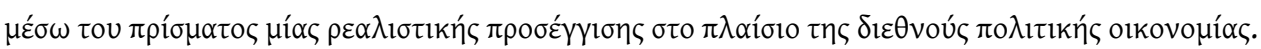

\section{INDEX}

Mots-clés : Grand Caucase, Grand Caucase, Union européenne, Union européenne, coopération régionale, coopération régionale, matières premières, matières premières, commerce, commerce, époque contemporaine, époque contemporaine

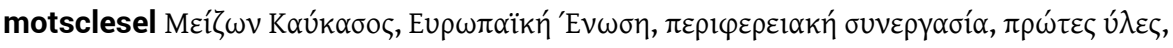

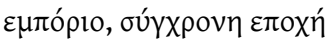

motsclestr Büyük Kafkasya, Avrupa Birliği, Bölgesel işbirliği, Hammadde ticareti, çağdaş dönem Keywords : Greater Caucasus, European Union, regional cooperation, raw materials, trade, contemporary period

\section{AUTEUR}

\section{ANASTASIA CH. LEKKA}

université du Pirée et université de Thessalie 